\title{
RESEARCH ASSAYS AND DIAGNOSTIC KITS
}

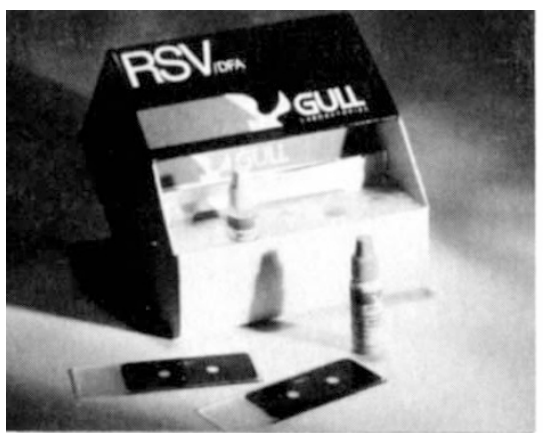

Respiratory Virus.

The RSV-MA $b$ test from Gull (Salt Lake City, UT) is intended for the direct detection of respiratory syncytial virus (RSV) in nasopharyngeal swab specimens, and in RSV isolated in cell culture. The kit features the RSV-MAb reagent, which contains murine monoclonal antibodies to the nucleoprotein and the fusion protein of RSV. The kit also includes mounting fluid and four RSV control slides. The positive well of the RSV control slides contains RSVinfected Vero cells, and the negative well contains uninfected cells. The test can be completed within 30 minutes of specimen preparation.

Write in 809 on Reader Service Card

\section{Immunostaining.}

Shandon Scientific (Runcorn, U.K.) introduces a compact workstation that standardizes and organizes immunostaining routines. The instrument organizes complex and lengthy immunostaining procedures involving reagent applications, incubations and washings, and offers complete protection to specimens. The slide and coverplate assembly has a three-sided well at the top to act as a reagent reservoir, and a channel through which reagent can pass. Staining is achieved by allowing a small amount of reagent placed in the well to be drawn by gravity down the channel and over the specimen.

Write in 806 on Reader Service Card

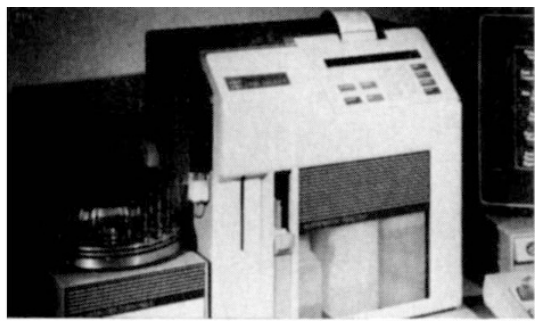

Biochemical Determination.

From Clandon Scientific (Farnborough, U.K.) comes the YSI 2700 SE-
LECT Biochemistry analyzer that offers a flexible approach to biochemical analysis for food and biotechnology laboratories. The system allows the operator to select from a range of nine chemistries, with variable sample size and adjustable calibration values. An immobilized enzyme electrode technology eliminates the problems of interference that affect traditional optical methods, providing results that are unaffected by color, turbidity, density, and temperature.

Write in 801 on Reader Service Card

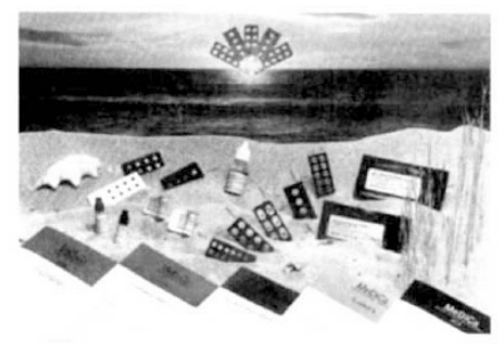

\section{IFA.}

MeDiCa (Carlsbad, CA) offers a variety of IFA test kits and substrate slides for clinical and research purposes. The kits come in sizes for 48, 96, and 192 tests. Four- and eight-well microscope slides are available with up to four different substrates per well. They will detect five or more autoantibodies in a single drop of patient serum by immunofluorescence.

Write in 813 on Reader Service Card

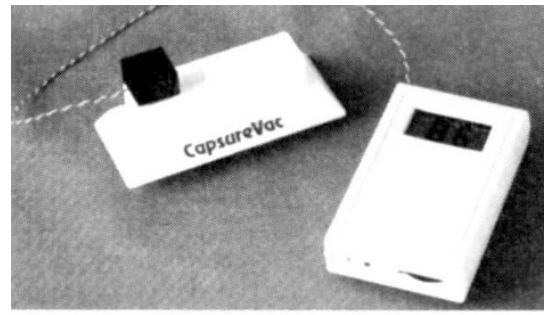

\section{Diagnostic Testing.}

Stratecon Diagnostics (Norwell, MA) introduces a solid phase format to develop immunoassays for rapid diagnosis with a quantitative determination. Up to four different membranes can be evaluated and compared side-by-side to find a match for the user's chemistry in a hand-held, portable, disposable format. Once R \& D is completed, the finished kit may be used in a doctor's office, or in any testing area that requires portability.

Write in 810 on Reader Service Card

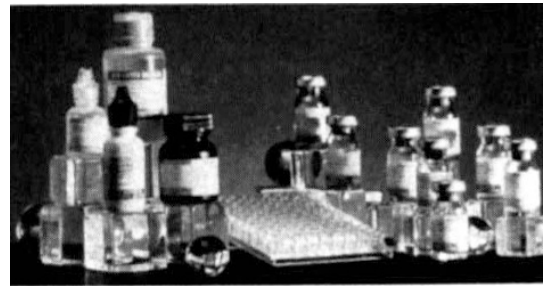

ELISA.

A wide range of ELISA test kits is offered by Medix Biotech (Foster City, $\mathrm{CA})$. The assaye use the 96-well microtiter plate format. Assays available for in vitro diagnostic use include $\mathrm{TSH}$, T3, T4, FT4, hCG total, Ferritin, HGH, and DHEA-S. Assays available for research use include U-TSH, CEA, Progesterone, Estradiol, Rubella, and CMV.

Write in 811 on Reader Service Card

\section{Tissue Extractor.}

The Homex 500 universal homogenizer-extractor from Bioreba (Basel, Switzerland) is designed for different analytical applications including seed, fruit, and food analysis. The apparatus is semi-automated, and is meant for use in routine or research laboratories in the fields of biological, zoological, and physiological sciences. Samples are contained within the homogenization bags throughout the sample processing, thereby eliminating carry-over of contaminants and the need for washing between samples. Typically about 300 samples per hour can be processed.

Write in 803 on Reader Service Card

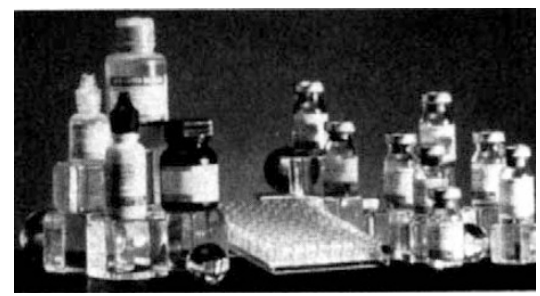

RheumELISA Plus.

From BioWhittaker (Walkersville, MD) come assays that use highly purified recombinant and naturally derived antigens to produce exceptional sensitivity and specificity. A major feature of the system is the ability to provide numerical results throughout the full dynamic range of the standard curve. The system includes assays for RNP, Sm, SS-A, and SS-B, and all are available for in vitro diagnostic use. The kits contain an antigen plate, and enough standards, controls, and reagents to perform 96 tests.

Write in 808 on Reader Service Card 